\title{
Study of Uninterruptible Duration Prediction Based on PC Operation
}

\author{
Hokuto Iga, Takahiro Tanaka, Kazuaki Aoki, and Kinya Fujita ${ }^{(凶)}$ \\ Graduate School, Tokyo University of Agriculture and Technology, 2-24-16 \\ Nakacho, Koganei, Tokyo 184-8588, Japan \\ 50014646103@st.tuat.ac.jp,kfujita@cc.tuat.ac.jp
}

\begin{abstract}
In order to manage interruptions adequately, the prediction of an office worker's uninterruptible duration is desired. We assumed three factors that may affect this uninterruptible duration: the type of work, the person's level of concentration, and the frequency with which the person is disturbed by others. For features related to the type of work, we adopted category of using application and determined the ratio of key-to-mouse usage. The rates of keystroke and mouse operation and the application-switching frequency were selected to reflect a person's concentration at work. A time of day was selected as an index which reflects the disturbance frequency. We then analyzed the relationship between these indices and the uninterruptible duration using 1200-h data. The results showed that, except for the time of day, a significant relationship exists between the uninterruptible duration and these indices. The combination of these indices appears promising for predicting the uninterruptible duration.
\end{abstract}

Keywords: Interruption $\cdot$ Prediction $\cdot$ Interruptibility $\cdot$ Uninterruptible duration $\cdot$ Work rhythm

\section{Introduction}

The frequency with which a person is interrupted while working has increased with the development of information systems. However, interruptions at inappropriate times may decrease a worker's productivity [1]. To address this concern, studies have been conducted to estimate user interruptibility and control of interruption timing. Estimating interruptibility has been attempted by assessing various worker conditions such as the worker's sitting position and the number of visitors received [2]; vital signals such as heart rate [3], pen usage, conversation status, and keystroke and mouse operation [4], and switching of applicationsoftware [5]. The combination of PC operation with the conversational status [6] or head motion [7] has also been examined as a means of improving the interruptibility estimation accuracy.

Furthermore, a remote awareness sharing system used in supporting the interruption judgment of a remote interrupter [8] and an automatic online communication mediating agent [9] have been developed. These systems allow an interrupter to recognize that a remote interruptee is not to be interrupted at the moment. However, these systems do not tell the interrupter how long he or she must wait until the interruptee can be 
interrupted. A solution for reconciling the conflicting needs of the interrupter and interruptee is to inform the interrupter of an expected waiting period. This method will allow the interrupter to perform work until the interruption time is reached, thus improving work efficiency. Simultaneously, this method prevents the interruptee from being interrupted while he or she is concentrating on the work. To achieve this, the prediction of interruptibility of a worker is required.

Several studies have been conducted on predicting future behavior of persons. For example, attempts to produce probabilistic predictions about a worker's return time to his or her office have been carried out on the basis of the personal history [10]. Additionally, modeling of work rhythm has been attempted [11]. However, predicting the duration in which a person cannot accept an interruption has not been studied. Therefore, this study examines the feasibility of predicting the uninterruptible duration of an office worker at a computer. Through experiments, this study assesses examined relationships between PC operation measures and the uninterruptible duration.

\section{Prediction of Uninterruptible Duration}

\subsection{Duration of PC Work}

Among studies on interruptibility estimation, methods employing PC-operation information have been found to be advantageous regarding the cost and implementation effort because they do not require setting up physical sensors in the work environment. Furthermore, prediction of uninterruptible duration, which a worker cannot accept an interruption, is desirable as mentioned in Sect. 1. Therefore, we defined the uninterruptible duration on the basis of the estimated interruptibility using PC-operation-based method [5]. The system estimates the "acceptable degree of interruption" at three levels: 1. Low, 2. Neither low nor high, and 3. High for every $500 \mathrm{~ms}$. The system prioritizes application switching (AS) moments over continuous working period, because breakpoint of the work is more suitable for interruption [12]. However, the increase of interruptibility associated with AS ends in a few seconds [13]. Therefore, this study adopts an interruptibility estimation algorithm for the not application switching period (NAS), which is based on four PC-operation indices, as shown in Fig. 1. In this study, we defined the "uninterruptible duration" as a period in which

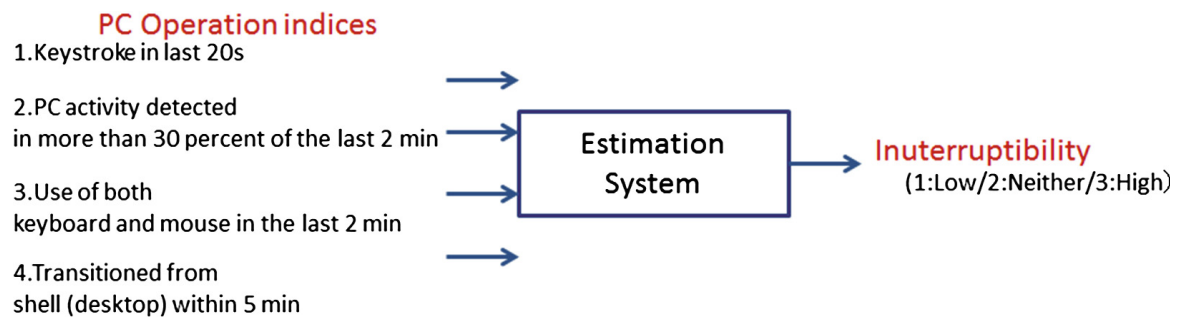

Fig. 1. Interruptibility estimation system 
the average interruptibility score for $1 \mathrm{~min}$ is less than 2.5 . The average score less than 2.5 represents that the estimated interruptibility was at most 2 for at least $30 \mathrm{~s}$ out of 1 min. Figure 2 illustrates the uninterruptible duration.

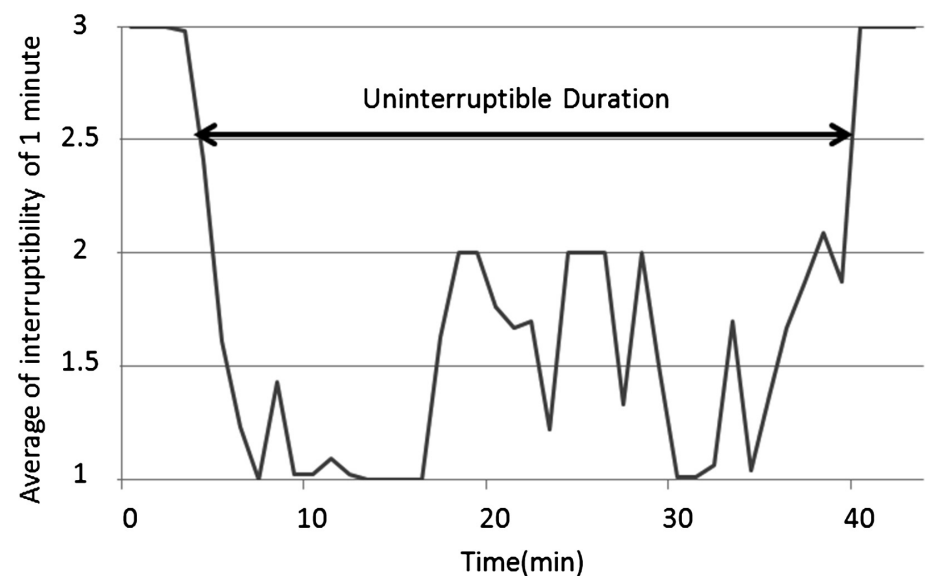

Fig. 2. Example of estimated interruptibility transition and definition of uninterruptible duration.

\subsection{Measures of Uninterruptible Duration}

Several factors are considered that affect the duration of work. We assumed three potential factors that affect the uninterruptible duration: the type of work, the worker's level of concentration, and the frequency of disturbance by others, as listed in Table 1.

Types of Work. Type of performing task might affect the uninterruptible duration. For example, Internet surfing will require less intellectual activity, then its uninterruptible duration to be short. In contrast, writing a document requires greater intellectual activity, we expect that uninterruptible duration will last longer. In addition, category of using application i.e., WEB browser, will have strong relationship with the task, i.e., Internet surfing. Thus, the types of work will be reflected on the category of using application software. Therefore, we have chosen the category of using application as a potential index of the uninterruptible duration.

Furthermore, uninterruptible durations vary even if a worker is using the same application. For example, we use a word processor for both document reading and

Table 1. Assumed factors affecting uninterruptible duration and examined indices

\begin{tabular}{l|l}
\hline Features & Indices \\
\hline \multirow{2}{*}{ Types of work } & category of using application software \\
\cline { 2 - 2 } & Ratio of mouse-to-key usage \\
\hline \multirow{2}{*}{ Concentration to work } & Rate of keystroke detection \\
\cline { 2 - 2 } & Rate of mouse-operation detection \\
\cline { 2 - 2 } & Application switching frequency \\
\hline Frequency of disturbance from others & Time of day \\
\hline
\end{tabular}


writing. Writing a document appears to require greater intellectual activity than reading a document. Moreover, document writing requires more keystrokes than does reading. Therefore, the ratio of mouse-to-key usage is considered as an index of uninterruptible duration.

Concentration at Work. If a worker loses concentration performing work because of internal factors such as fatigue, he or she will gradually lose focus on the task at hand. In addition, this lack of focus will induce a change in physical activity such as a decrease in the number of keystrokes. Therefore, measurements of the keystroke and mouse operation detection rates and application-switching frequency are assumed to predict the uninterruptible duration.

Frequency of Disturbance by Others. Disturbance by others is impossible to predict because it is an outside factor from a disturbed worker. However, the chance of disturbance, such as from visitors, will vary depending on time of day. Therefore, we hypothesized that a time of day has a relationship with the uninterruptible duration.

We then analyzed the relationship between the uninterruptible duration and the six selected indices, which are related to the previously mentioned three factors.

\section{Analysis of Uninterruptible Duration}

\subsection{Analyzed Data}

In order to analyze the relationship between the uninterruptible duration and PC operations, we recorded the PC operations of workers and automatically estimated the interruptibility levels as they worked. The experiment is shown in Fig. 3. We recruited four participants: two university students and two faculty members. The participants performed ordinary research and miscellaneous activities without any restriction on tasks to perform. The average recording time of PC operational record per day was approximately $10 \mathrm{~h}$. The number of recorded days for a month varies with the participants from 10 to 20 days. The entire data set of PC operation consists of 1200-h. The individual recorded periods were three and two months for each student and each faculty member, respectively. The PC operation records include the system time, the estimated interruptibility, the name of application used primarily, and the keystroke and mouse usage. The activities observed most were: document writing, data analysis, programming, and internet surfing. Disturbances by others were occasionally observed.

The distribution of the recorded uninterruptible durations is shown in Fig. 4. Durations less than 3 min were excluded because predicting when these short tasks would end was unnecessary. In addition, we assumed that at least 3 min of PC-operation information is necessary for prediction. The number of uninterruptible durations exceeding $3 \mathrm{~min}$ was 1819 . The average duration was $15.4 \mathrm{~min}$. The most frequently observed duration was between 4 and $6 \mathrm{~min}$. The number of observed uninterruptible durations exhibited a tendency to decrease with the duration. We also observed several long uninterruptible durations greater than $40 \mathrm{~min}$. The application software primarily used by faculty members were web browsers and software development tools known as Visual Studio. Longer uninterruptible durations took place 


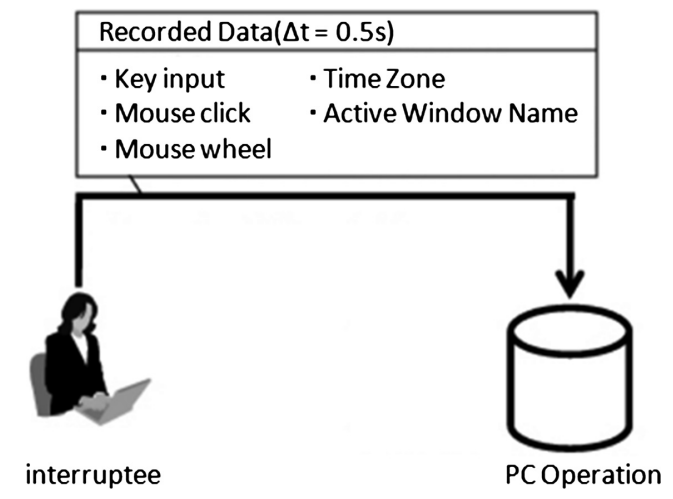

Fig. 3. Experimental system for collecting records for analysis

more frequency at night. The main application software used by students were web browsers and the Excel spreadsheet program. Students performed these activities primarily in the afternoon.

\subsection{Method of Analysis}

We clustered the uninterruptible durations on the basis of the six indices discussed in Sect. 2.2.

The using application software for each uninterruptible period is defined as the application whose total focus time is the longest among the used software. The applications used mostly were: Explorer, web browser, Acrobat Reader, Word, Excel, and Visual Studio.

The keystroke detection rate is determined on the basis of the proportion of the keystroke detection time during the uninterruptible duration. Here, the uninterruptible durations might be different among superficially similar activities such as writing business documents, user's manuals, and research manuscripts. Moreover, the differences in duration may be related to the keystroke detection rate. Therefore, we divided the keystroke detection rate into six percentage ranges: $0-5 \%, 5-10 \%, 10-15 \%, 15-20 \%$, 20-30, and $>30 \%$.

The mouse-operation detection rate is defined as the proportion of mouse click or wheel usage time to the uninterruptible duration. We divided the mouse-operation detection rate into three percentage ranges: $0-5 \%, 5-10 \%$, and $>10 \%$. We speculate that the uninterruptible duration of work having a high mouse-operation detection rate, such as when surfing the Internet, is shorter.

The rate of mouse usage is the proportion of the mouse-operation detection time to the keystroke detection time. We divided this rate into five ranges: $0-0.5,0.5-1.0$, $1.0-1.5,1.5-2.0,>2.0$. A rate close to zero means that the worker is mainly using the keyboard. A value greater than one represents tasks mainly using mouse. We expect that tasks mainly using a mouse require less intellectual activity, and their uninterruptible durations are short. 


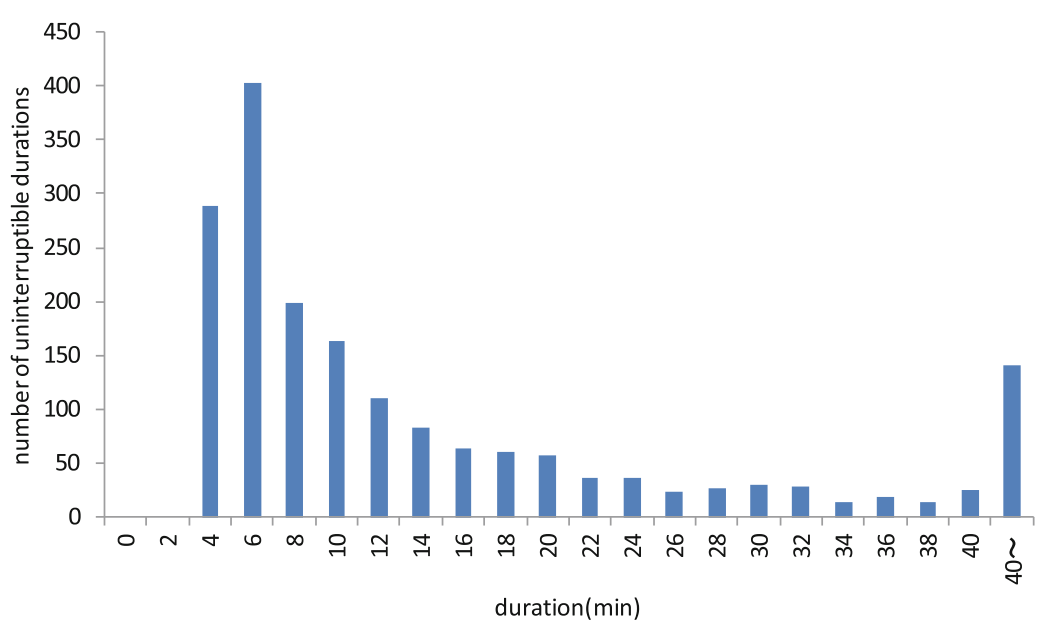

Fig. 4. Distribution of uninterruptible durations

The AS frequency is defined as the average switching frequency per minute. However, it is obviously difficult to work using application software, which is only focused on for few seconds. Furthermore, a previous study reported that it requires more than $2 \mathrm{~s}$ to start working after a task transition [13]. Therefore, we omitted applications, which are focused on for less than $2 \mathrm{~s}$. The AS frequency was divided into four levels: $0-1,1-2,2-3,>3$. When the detected AS frequency is less than one, the worker is considered to be mainly using one software application. When the application-switching frequency exceeds three, it indicates that the worker is using multiple software applications for a task.

We defined a time of day as the time when the uninterruptible period starts, which is the time when the 1-min average interruptibility fell below 2.5. Because the frequency of interruption from other persons could be different in the morning, afternoon, and evening, we divided the time of day into four levels: before 12:00, 12:00-15:00, 15:00-18:00, and after 18:00. It is speculated that interruptions from others occur more often in the afternoon and less in the evening.

We calculated the above-mentions six indices for each detected uninterruptible duration, which represents a unit of concentrated work. Then, we calculated the average uninterruptible durations for each cluster of each index.

\subsection{Results}

Figure 5 shows the average uninterruptible durations for each cluster of the six indices. The dotted line represents the average value for all durations.

Category of using application. From the result, the uninterruptible durations were significantly different depending on the using application software. The uninterruptible duration was especially shorter when the interruptee was using a web browser. On the other hand, the duration became longer when the worker was mainly using Excel or 


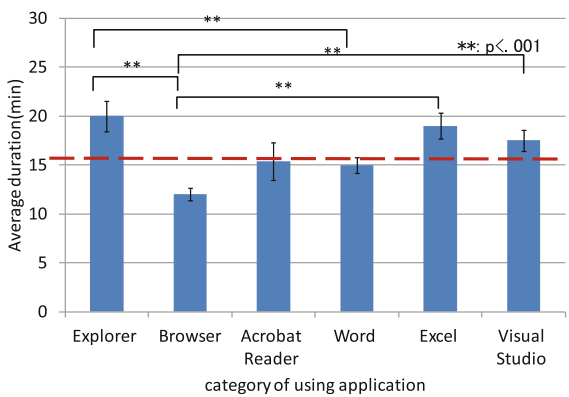

(a)

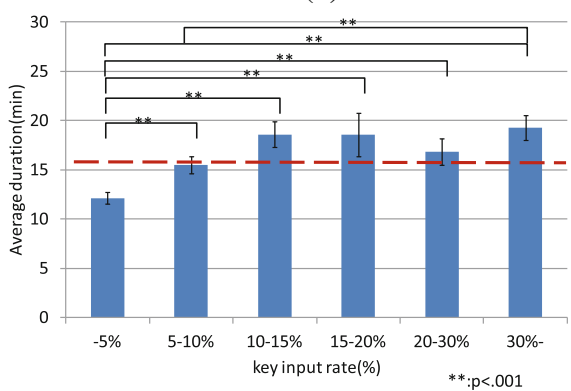

(c)

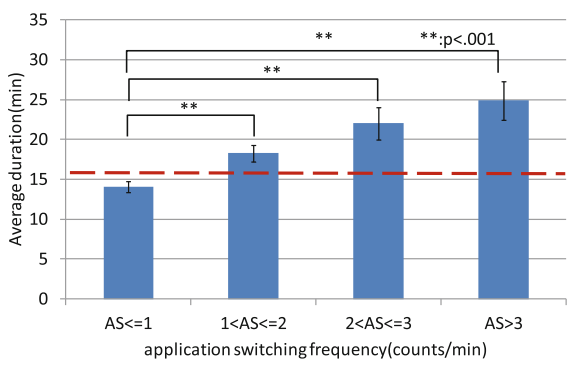

(e)

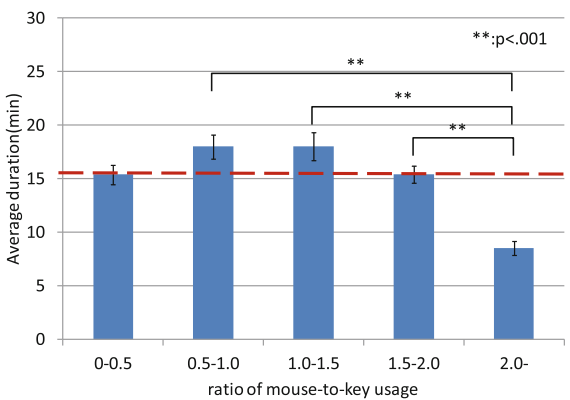

(b)

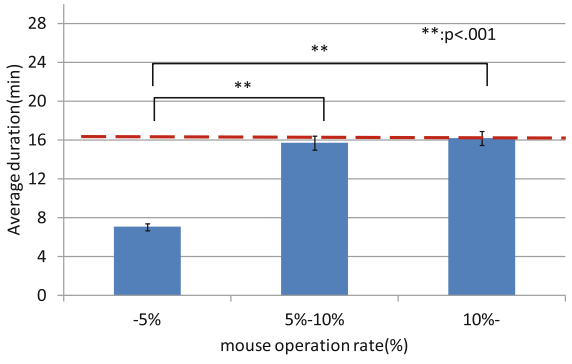

(d)

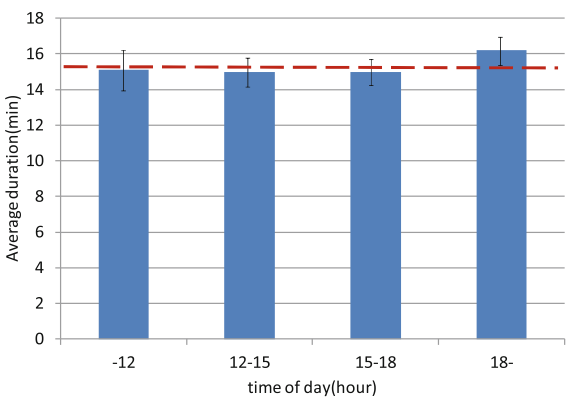

(f)

Fig. 5. Average uninterruptible durations for clusters of each feature: (a) category of using application, (b) ratio of mouse-to-key usage, (c) keystroke detection rate, (d) mouse-operation detection rate, (e) application-switching frequency, and (f) time of day.

Visual Studio. The longer lasting uninterruptible durations for the data analysis and programming suggest their larger task size in terms of intellectual activity requirements compared to web browsing.

Ratio of Mouse-to-Key Usage. Basically, a significant tendency is observed in which the uninterruptible durations decrease with the increase in the mouse-to-key usage ratio. This coincides with the expectation that $\mathrm{PC}$ operations using a mouse require less intellectual activity than text writing using a keyboard. Interestingly, it was also 
suggested that the uninterruptible duration was shorter when the time spent for mouse-operation was less than half for mouse operation.

Keystroke Detection Rate. As seen in Fig. 5(c), the uninterruptible duration tends to be longer when a worker is engaged in tasks requiring more keystrokes, as expected. Durations with a keystroke detection rate of less than $5 \%$ were significantly shorter than the others.

Mouse Operation Detection Rate. The duration of work with a lower mouse-operation rate was short, similar to the keystroke detection rate. In particular, the average duration for work for a mouse-operation rate of $5 \%$ or less was 7 min and significantly shorter than the other cases.

Application-Switching Frequency. In Fig. 5(e), an increase in the uninterruptible duration with the AS frequency is observed. It is speculated that the AS frequency reflects the degree of engagement of a worker in a task. It is also suggested that the durations for tasks less than or equal to an AS frequency of $1 \mathrm{~min}^{-1}$ are significantly shorter than the others.

Time of day. The uninterruptible durations during the evening were slightly longer than those during the day. This is in agreement with our assumption that there are fewer meetings and visitors in the evening, thereby making the uninterruptible durations longer. The durations in the morning were also slightly longer than those in the afternoon.

These results demonstrated that the average uninterruptible durations vary with the situation. The clusters based on the category of using application, the ratio of mouse-to-key usage (proportional to key usage), the keystroke detection rate, the mouse-operation detection rate, and the AS frequency exhibited significant relationships with the uninterruptible duration. These results suggest the possibility of predicting the uninterruptible duration by combining these indices.

\section{Discussion}

The average uninterruptible duration of all work was $15.4 \mathrm{~min}$, as described in Sect. 3. The duration for editing a particular page in Wikipedia was $15 \mathrm{~min}$ [14] in the previous study. Therefore, the observed average uninterruptible duration in this study appears reasonable in terms of the continuous duration of a single task.

Additionally, we confirmed the variation in the uninterruptible duration with each index, as shown in Sect. 3. The observed differences almost fulfilled our expectation, such that the uninterruptible duration varies within the indices. However, the uninterruptible durations were longer, mainly when using Explorer, even though we expected the duration to be short. This is because an interruptee works using multiple files or applications through Explorer. Furthermore, the work duration when Word was mainly used was thought to be longer because the worker will concentrate on document writing. However, the average uninterruptible duration when a user was mainly using Word was less than the entire average of the uninterruptible duration. This result suggests that the interruptee uses Word for tasks requiring a higher intellectual load 
(e.g., writing manuscripts) and for tasks with a lower intellectual load (e.g., document viewing). For the ratio of mouse-to-key usage, we expected to confirm a longer duration when keystrokes are frequently detected. However, the durations when the ratio is within 0.5 to 1.5 (i.e., the key usage level and the mouse usage level were approximately the same) were the longest. We also considered that a ratio less than 0.5 includes data with little PC usage. For example, if a user had 10 keystrokes and two mouse clicks, it was categorized as a ratio less than 0.5 , even though the uninterruptible duration is short.

In future studies, it is necessary to define and analyze the effects of other features such as combination of the currently used application. We consider that the inclusion of a secondary application in the analysis will provide more information on the nature of the work that the user is engaged in. For example, a user may use a web browser and editor at a same time while collecting information.

The prediction of the uninterruptible duration is also a future challenge. It is necessary to establish a mathematical prediction model of the uninterruptible duration based on the indices analyzed in this study. Modeling techniques in addition to ordinary multiple regression analysis, e.g., mathematical quantification theory class I, are needed to establish an adequate expression for a mathematical prediction.

\section{Conclusion}

In this study, we analyzed the relationship between the uninterruptible duration and six indices on the basis of 1200-h PC-operation data recorded from four participants. The indices - the category of using application, the keystroke detection rate, the mouse-operation detection rate, the ratio of mouse-to-key usage, and the AS frequency - demonstrated significant relationships with the uninterruptible duration. We also confirmed that the uninterruptible duration possibly depends on the time of day. The next step is to establish a prediction model for the uninterruptible duration on the basis of the discussed indices.

Acknowledgements. This work was partly supported by Kakenhi from JSPS, the fund for smart space technology toward a sustainable society from MEXT, and the fund for an ultrarealistic communication system from NICT.

\section{References}

1. Bailey, B.P., Joseph, A.K., John, V.C.: The effect of interruptions on task performance, annoyance, and anxiety in the user interface. Proc. INTERACT 1, 593-601 (2001)

2. Hudson, S.E., Fogarty, J., Atkeson, C.G., Avrahami, D., Forlizzi, J., Kiesler, S., Lee, J.C., Yang, J.: Predicting human interruptibility with sensors: a wizard of oz feasibility study. In: Proceedings of SIGCHI, pp. 257-264 (2003)

3. Chen, D., Hart, J., Vertegaal, R.: Towards a physiological model of user interruptibility. Proc. INTERACT, Part 2, 439-451 (2007) 
4. Minakuchi, M., Takeuchi, T., Kuramoto, I., Shibuya, Y., Tsujino, Y.: An automatic estimation method for busyness at desktop. J. Hum. Interf. Soc. 6(1), 69-74 (2004). (in Japanese)

5. Tanaka, T., Fujita, K.: Study of user interruptibility estimation based on focused application switching. In: Proceedings of CSCW, pp. 721-724 (2011)

6. Hashimoto, S., Tanaka, T., Aoki, K., Fujita, K.: improvement of interruptibility estimation during PC work by reflecting conversation status. IEICE Trans. Inf. Syst. 97(12), 3171-3180 (2014)

7. Tanaka, T., Abe R., Aoki, K., Fujita, K.: Interruptibility estimation based on head motion and PC operation. Int. J. Hum.-Comput. Interact. (to appear)

8. Bjelica, M.Z., Nrazovac, B., Papp, I., Teslic, N.: Context-aware platform with user availability estimation and light-based announcement. IEEE Trans. Syst., Man, Cybern.: Syst. 43, 1228-1239 (2013)

9. Tanaka, T., Fujita, K.: Interaction mediate agent based on user interruptibility estimation. In: Smith, M.J., Salvendy, G. (eds.) HCII 2011, Part I. LNCS, vol. 6771, pp. 152-160. Springer, Heidelberg (2011)

10. Yamagoe, K., Kuzuoka, H.: Developing of a meeting support system based on work rhythm. In: Proceedings of the Human Interface Symposium, pp. 741-744 (2003) (in Japanese)

11. Begole, J.B., Tang, J.C., Smith, R.B., Yankelovich, N.: Work rhythms: analyzing visualizations of awareness histories of distributed groups. In: Proceedings of CSCW, pp. 334-343 (2002)

12. Iqbal, S.T., Bailey, B.P.: Effects of intelligent notification management on users and their tasks. In: Proceedings of SIGCHI, pp. 93-102 (2008)

13. Tanaka, T., Fujita, K.: Discussion on duration of uninterruptiblity reduction at focused application-switching. J. Japan Soc. Fuzzy Theory Intell, Inform. 21(5), 827-836 (2009). (in Japanese)

14. Geiger, R.S., Halfaker, A.: Using edit sessions to measure participation in wikipedia. In: Proc. CSCW, pp. 861-870 (2013) 